\title{
Sex Differences in Fetal Rabbit Pulmonary Surfactant Production
}

\author{
HEBER C. NIELSEN ${ }^{(2.5)}$ AND JOHN S. TORDAY \\ Department of Pediatrics, Harvard Medical School, Boston, Massachusetts, USA
}

\begin{abstract}
Summary
Male infants have a higher risk of respiratory distress syndrome than females at concurrent gestations. Recent evidence in humans has linked fetal sex with differences in amniotic fluid indices of lung maturation. We tested the hypothesis that the late gestation surge in pulmonary surfactant production occurs later in the male fetus than in the female fetus in the rabbit model. We measured saturated phosphatidylcholine and total phosphatidylcholine in lung lavage at 26,28 , and 30 days gestation and in amniotic fluid at $24,26,28$, and 30 days gestation (term $=31$ days). The saturated phosphatidylcholine/sphingomyelin ratios were 158 and $55 \%$ higher in female fetal lung lavage at 26 and 28 days, respectively, and $75 \%$ higher in amniotic fluid at 28 days $(P<0.05)$. The total phosphatidylcholine/sphingomyelin ratios were $39 \%$ higher in female fetal lung lavage and $35 \%$ higher in female amniotic fluid at 28 days $(P<0.05)$. Significant differences were not detected in the very immature ( 24 day) or the mature ( 30 day) fetuses. This provides clear evidence of a biochemical difference according to fetal sex in the maturation of pulmonary surfactant production.
\end{abstract}

\section{Speculation}

This is the first documentation of a biochemical difference according to fetal sex arising in the course of normal fetal development which is directly related to an increase in morbidity and mortality in the newborn. Further investigations using this model may help to define the mechanism by which the sex difference in fetal lung maturation occurs. This may lead to development of better methods for preventing respiratory distress syndrome in the premature infant. Understanding the causes of the male disadvantage for respiratory distress syndrome may also help to define the causes of the male disadvantage for a variety of other disorders.

Males are at greater risk than females for a variety of diseases and have higher age-specific mortality rates, a phenomenon termed the male disadvantage. The respiratory distress syndrome (RDS) of the newborn is an example of the male disadvantage. Male newborns are at an increased risk of developing RDS at equivalent points in gestation and have a 1.7 -fold higher death rate from RDS than do female newborns $(14,16,22)$.

RDS is related to an insufficient quantity of pulmonary surfactant, usually in association with premature birth. The bulk of pulmonary surfactant is phosphatidylcholine (PC), and the principle surface-active component is saturated (usually dipalmitoyl) phosphatidylcholine (SPC). The attainment of lung maturity in late gestation is characterized by a marked increase in production of total PC, particularly of SPC (4).

We hypothesized that the sex difference in RDS is related to a delay in the male fetus of the late gestation surge in pulmonary surfactant production. We tested this hypothesis by measuring PC and SPC in lung lavage (LL) and amniotic fluid (AF) in the fetal rabbit at $24,26,28$, and 30 days gestation.

\section{MATERIALS AND METHODS}

New Zealand White rabbit does were mated between 0900 and $1200 \mathrm{hr}$ and sacrificed between these hours on day $24,26,28$, or 30 of gestation (day $0=$ day of mating; term $=31$ days). Does were sacrificed with an intravenous injection of pentobarbital (180 $\mathrm{mg}$ ). The uterus was then exposed, and the fetuses were sacrificed with $30 \mathrm{mg}$ of pentobarbital intraperitoneally, injected through the uterine wall. AF was obtained on each fetus by puncturing the fetal membranes after dissecting away the uterine wall. Each fetus was weighed and then tracheostomized, and LL was obtained by lavaging the lungs with $5 \times 0.5 \mathrm{ml}$ of $0.9 \%$ iced $\mathrm{NaCl}$ solution. We were unable to obtain reliable LL samples on 24 day gestation fetuses. Bloody LL or AF samples were discarded. Fetal sex was determined by inspection of the gonads and confirmed histologically.

Phospholipids were extracted from the AF and LL by the method of Folch et al. (5) and separated by thin-layer chromatography. SPC was isolated by the method of Mason et al. (13) as modified by Torday et al. (19). The individual phospholipid fractions [i.e., PC, SPC, and sphingomyelin (S)] were quantitated by chemical assay of inorganic phosphorus (2). S concentration remains relatively constant in LL and AF during late gestation (8) and is widely used as an internal standard to avoid the technical problems of obtaining quantitative samples of AF or LL. Therefore, the results are expressed as the ratio of PC or SPC to S (PC/ $\mathrm{S}$ and SPC/S ratios).

All statistical comparisons were made using Student's $t$ test.

\section{RESULTS}

There were no differences between the mean male and female body weights at each point in gestation studied (data not shown). Figure 1 shows the results for the $\mathrm{PC} / \mathrm{S}$ and $\mathrm{SPC} / \mathrm{S}$ ratios for both sexes in $\mathrm{LL}$ and $\mathrm{AF}$ at each point in gestation. In the SPC/S ratio, there is a significant difference between the males and females characterized by a later rise in the SPC/S ratio in the male fetus. This difference appears only in the LL at 26 days and in both LL and $\mathrm{AF}$ at 28 days. The female SPC/S ratio is $158 \%$ higher than the male SPC/S ratio in the LL at 26 days, and at 28 days, the female SPC/S ratio is 55\% higher than the male in the LL and 75\% higher in the AF. At 30 days, there was no observable sex difference in LL or AF. A test for type II error in the AF at 30 days showed that more than 200 fetuses in each group would be needed to achieve statistical significance.

In the $\mathrm{PC} / \mathrm{S}$ ratio, sex differences were less striking and were only apparent on the 28 th day. The female PC/S ratio was $39 \%$ higher in the LL and 35\% higher in the AF. At 30 days, there were no detectable differences.

\section{DISCUSSION}

Our data demonstrate directly for the first time that there is a sex difference in fetal pulmonary surfactant production during 

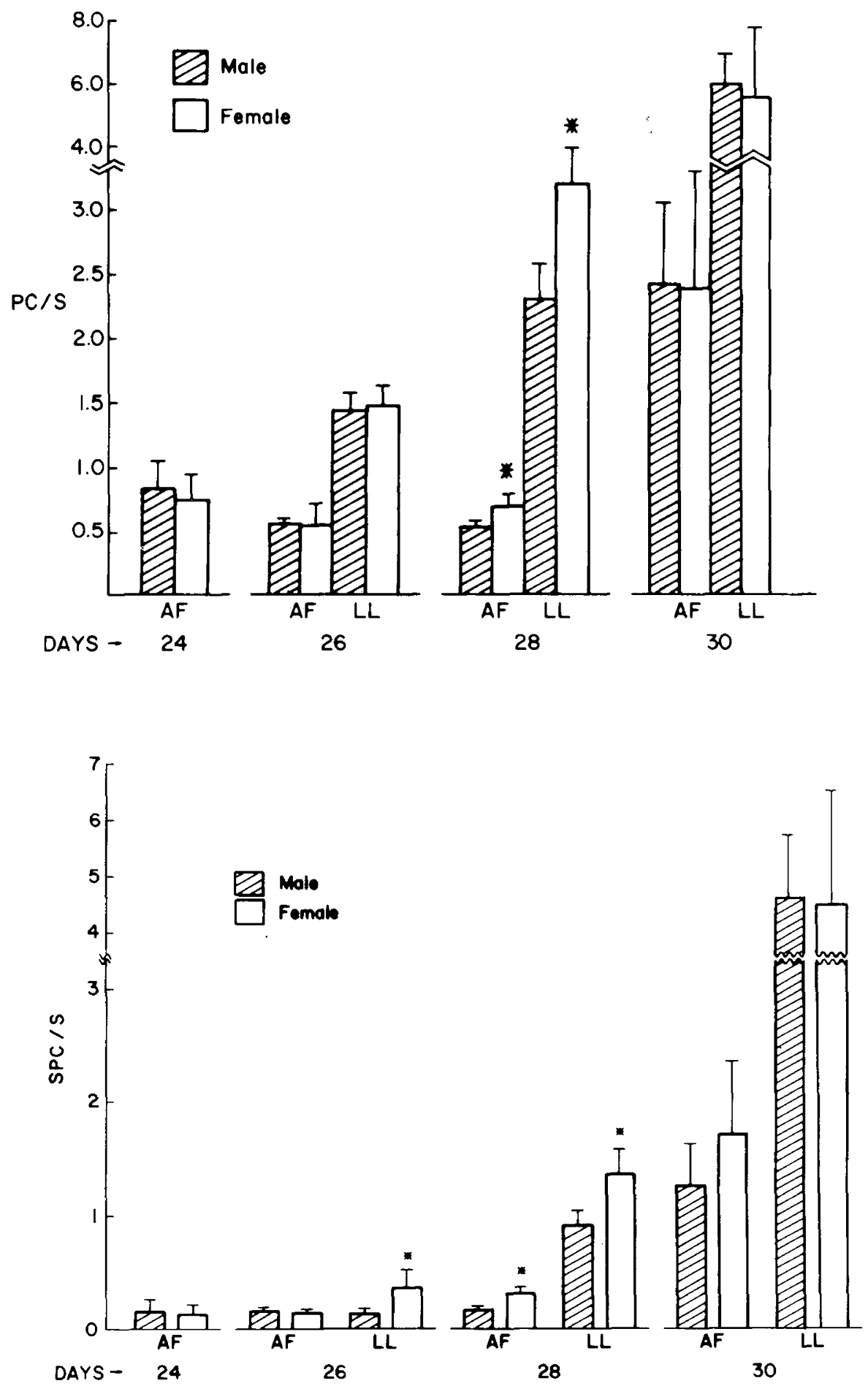

Fig. 1. Developmental profile of the production of PC, shown as PC/S ratio (upper panel) and SPC shown as $S P C / S$ ratio (lower panel) by male and female rabbits in AF and LL. Bars, mean \pm S.E., ${ }^{*}, P<0.05$ by Student's $t$ test. Three litters were studied at each gestational age, with the following numbers of fetuses: 24 days gestation: 11 males, 12 females; 26 days gestation: LL, 14 males, 11 females; AF, 12 males, 11 females; 28 days gestation: LL, 13 males, eight females; AF, 11 males, eight females; 30 days gestation: LL 17 males, six females; AF, 15 males, six females.

late gestation. Previous studies had provided indirect evidence for this. We had analyzed a number of human amniotic fluid indices of fetal lung maturation (i.e., L/S ratio and SPC and cortisol concentration) and found significantly lower levels in male versus female fetuses during the last trimester (15). Kotas and Avery (12) recently reported a sex difference in the pressure-volume characteristics of 27 day fetal rabbit lungs. Males had less deflation stability than did females. Taken together, these findings suggest that male newborns are at greater risk of RDS morbidity and mortality because as a group there are more male than female fetuses producing insufficient quantities of pulmonary surfactant at concurrent points in gestation.

Clinically, physicians use measurements of PC or SPC in amniotic fluid to assess the risk of RDS during late gestation $(7,19)$. The threshold of "maturity" is very discrete for these measurements. Values of PC or SPC which are below the threshold of maturity indicate a significant increase in risk of $\operatorname{RDS}(7,19)$. The timing of pulmonary surfactant production in the fetal rabbit is similar to that in humans (18). RDS can be created in the rabbit by premature delivery. At 28 days, one-half of the rabbit pups 
will die of hyaline membrane disease (1), making this stage in rabbit lung maturity roughly equivalent to $28 \mathrm{wk}$ gestation in humans (4). Although we have not provided physiologic evidence for increased risk of RDS in male rabbits, we believe that the observed biochemical differences at 26 and 28 days reflect such a difference.

There are several possible mechanisms through which a sex difference in fetal metabolism might occur. We are focusing our attention on possible hormonal mechanisms because fetal lung development is under hormonal regulation (4). Recently, the effect of gonadal hormones, particularly androgens, on the development of nonreproductive organs such as brain, liver, and adrenal cortex has been a topic of investigation $(3,10,11)$. Testosterone levels are higher in male fetuses than in female fetuses (6), and in rabbits and rats the difference is greatest just prior to the time when the surge in pulmonary surfactant production has been shown to begin $(20,21)$. Hence, testosterone may be involved in the delayed timing of pulmonary surfactant maturation of the male. On the other hand, Khosla and Rooney (9) and Sarda et al. (17) have shown that estradiol stimulates the production of SPC in fetal rabbit lung and tissue culture, respectively. However, it is unclear how estrogens would produce the observed sex difference in lung maturation because fetal estrogen levels are similar for male and female fetuses throughout gestation (6). Further investigation using this model may help to define how the sex difference in fetal lung maturation is mediated and, more specifically, to define the hormonal regulation of fetal pulmonary surfactant production.

\section{CONCLUSION}

The male disadvantage for RDS is related to a delay in the maturation of pulmonary surfactant production in the male fetus. This occurs as a process of normal fetal development. Further studies are needed to elucidate the mechanism for this sex difference.

\section{REFERENCES AND NOTES}

1. Barrett, C. T., Sevanian, A., Phelps, D. L., Gilden, C., and Kaplan, S. A.: Effects of cortisol and aminophylline upon survival, pulmonary mechanics and secreted phosphatidylcholine of prematurely delivered rabbits. Pediatr. Res., 12: 38 (1978).

2. Bartlett, G. R.: Phosphorus assay in column chromatography. J. Biol. Chem., 234: 466 (1959)

3. DeMoor, P., Verhoeven, G., Lamberigts, G., and Heyns, W.: Organizing effects of "early" testosterone secretion on steroid metabolism and binding. In: V. H T. James, M. Serio, and L. Martini: Endocrine Function of the Human Testes. pp. 343-369 (Academic Press, Inc., New York, 1973).
4. Farrell, P. M., and Avery, M. E.: Hyaline membrane disease. Am. Rev. Respir. Dis., 3: 657 (1975).

5. Folch, J., Lees, M., and Sloane-Stanley, G. H.: A simple method for the isolation and purification of total lipids from animal tissues. J. Biol. Chem., 226: 497 (1957).

6. Forest, M. G.: Function of the ovary in the neonate and infant. Eur. J. Obstet. Gynec. Reprod. Biol., 9: 145 (1979).

7. Gluck, L., Kulovich, M. V., Borer, R. C., Brenner, P. H., Anderson, G. G., and Spellacy, W. N.: Diagnosis of the respiratory distress syndrome by amniocentesis. Am. J. Obstet. Gynecol., 109: 440 (1971).

8. Gluck, L., Scribney, M., and Kulovich, M. V.: The biochemical development of surface activity in mammalian lung. II. The biosynthesis of phospholipids in the lung of the developing rabbit fetus and newborn. Pediatr. Res., 1: 247 (1967).

9. Khosla, S. S., and Rooney, S. A.: Stimulation of fetal lung surfactant production by administration of 17 beta-estradiol to the maternal rabbit. Am. J. Obstet. Gynecol., 133: 213 (1979).

10. Kitay, J. I.: Effects of estrogen and androgen on the adrenal cortex of the rat. In K. W. McKerns: Function of the Adrenal Cortex. pp. 775-810 (AppletonCentury-Crofts, New York, 1968).

11. Kolata, G. B.: Sex hormones and brain development. Science (Wash. D. C.), 205: 985 (1979).

12. Kotas, R. V., and Avery, M. E.: The influence of sex on fetal rabbit lung maturation and on the response to glucocorticoid. Am. Rev. Respir. Dis., 121: 377 (1980).

13. Mason, R. J., Nellenbogen, J., and Clements, J. A.: Isolation of disaturated phosphatidylcholine with osmium tetroxide. J. Lipid Res., 17: 281 (1976).

14. Miller, H. C., and Futrakul, P.: Birthweight, gestational age and sex as determining factors in the incidence of respiratory distress syndrome of prematurely born infants. J. Pediatr., 72: 628 (1968).

15. Nielsen, H. C., Torday, J. S., Fencl, M., and Avery, M. E.: Sex differences in human fetal lung maturation. Am. Rev. Respir. Dis., 123: 205 (1981).

16. Robert, M. F., Neff, R. K., Hubbell, J. P., Taeusch, H. W., and Avery, M. E.: Maternal diabetes and the respiratory distress syndrome. N. Engl. J. Med., 294: 357 (1976).

17. Sarda, I. R., Hayglass, K., Gorwill, R. H., and Smith, B. T.: Estrogens and lung maturation: dual effect on rabbit fetal lung cell culture. Proceedings of the Endocrine Society, p. 90 (1980).

18. Spanner, S.: Separation and analysis of phospholipids, In: G. B. Ansell, N. J. Hawthorne, and R. M. C. Dawson: Form and Function of Phospholipids. pp. 43-65 (Elsevier Scientific Publishing Co., New York, 1973).

19. Torday, J. S., Carson, L., and Lawson, E. E.: Saturated phosphatidylcholine in amniotic fluid and prediction of the respiratory distress syndrome. N. Engl. J. Med., 301: 1013 (1979).

20. Veyssiere, G., Berger, M., Jean-Faucher, C., DeTurckheim, M., and Jean, C.: Levels of testosterone in the plasma, gonads, and adrenals during fetal development of the rabbit. Endocrinology, 99: 1263 (1976).

21. Ward, I. L., and Weisz, J.: Maternal stress alters plasma testosterone in fetal males. Science (Wash. D. C.), 207: 328 (1980).

22. Wood, R. E., and Farrell, P. M.: Epidemiology of respiratory distress syndrome (RDS). Pediatr. Res., 8: 452 (1974).

23. Dr. Nielsen is a Parker B. Francis fellow in pulmonary research.

24. Dr. Torday is the recipient of a grant of the King Trust Foundation.

25. Requests for reprints should be addressed to: Heber C. Nielsen. M.D., Joint Program in Neonatology, Boston Hospital for Women, 221 Longwood Avenue, Boston, MA. 02115 (USA).

26. Received for publication October 8, 1980.

27. Accepted for publication February 2, 1981. 